\title{
Gas fiber lasers may represent a breakthrough in creating powerful radiation sources in the mid-IR
}

\author{
Andrey Pryamikov ${ }^{1 凶}$
}

\begin{abstract}
Continuous wave fiber laser created on the basis of silica glass negative curvature hollow core fiber filled with $\mathrm{HBr}$ make it possible to obtain efficient narrow linewidth mid-IR emission with a maximum laser power of about $500 \mathrm{~mW}$ at wavelength of $4200 \mathrm{~nm}$. It is for the first time that emission from a continuous wave fiber laser have been achieved at a wavelength of $4496 \mathrm{~nm}$ with the largest tuning range of $686 \mathrm{~nm}$.
\end{abstract}

It is well known that creation and development of discrete and tunable sources of the mid - IR laser radiation has a very long history. The basis of mid-IR photonics was laid when creating quantum cascade lasers ${ }^{1}$, vibronicstate lasers ${ }^{2}$ and optical parametric oscillators ${ }^{3}$. All these types of solid- state mid-IR lasers have their advantages and disadvantages. For example, quantum cascade lasers which were built out of quantum semiconductor structures can cover the spectral range from the mid-infrared to the sub-millimiter spectral region in the continuous wave $(\mathrm{CW})$ mode. But, the heat issue generates serious challenges for creating of high-power mid-infrared light sources. Although the other above-mentioned solid-state laser sources in the mid-IR spectral range have an efficiency sufficient for commercial applications, significant disadvantages ${ }^{4}$ such as a narrow linewidth and linear polarized excitation for parametric generation are still widely exists.

Over the last decade, the development of high-power fiber lasers operating in the mid-infrared spectral range has grown massively, partly thanks to a rapidly maturing technology of soft-glass fiber fabrication ${ }^{5}$. Optical fibers made of soft glasses have significantly lower phonon absorption in the mid-IR spectral range compared to optical fibers made of silicate glass or rare-earth-doped silica glass fibers and, accordingly, significantly lower material losses ${ }^{6}$. This factor makes it possible to actively

\footnotetext{
Correspondence: Andrey Pryamikov (pryamikov@mail.ru)

${ }^{1}$ Prokhorov General Physics Institute of the Russian Academy of Sciences, 38 Vavilov street, Moscow, Russia
}

use them to create power fiber lasers in the mid-IR ${ }^{4}$. Fiber lasers based on fluoride glasses have proved particularly successful $^{7}$. For example, erbium - doped fluorozirconate glass fibers allowed to generate 30 Watt mid-infrared laser output power at a wavelength near $3 \mu \mathrm{m}^{8}$ and watt-level fiber laser output power at wavelengths up to $3.55 \mu \mathrm{m}^{9}$ (Fig. 1). The longest wavelength generation in the mid-IR from the fluoride fiber laser was obtained at a wavelength of $3.92 \mu \mathrm{m}$ using a heavily holmium-doped fluoroindate fiber at CW output power of $200 \mathrm{~mW}^{7}$ (Fig. 1). Despite such an impressive result, since then no new record values of $\mathrm{CW}$ output power at wavelengths at and beyond of $4 \mu \mathrm{m}$ have been obtained in fluoride fiber lasers which is primarily due to high quantum defect, thermal management and fiber failure ${ }^{10}$.

The situation in the field of fiber lasers operating in the mid-IR spectral range began to change dramatically with the development of hollow core fiber technology. This was especially evident after the creation of the so called "negative curvature" or "anti-resonant" hollow core fibers ${ }^{11-13}$ (Fig. 2). They had promising optical properties which allowed to transmit light in the mid-IR spectral range even in fibers made of silica glass ${ }^{14}$. The transmission spectrum of these hollow core fibers with a cladding consisting of capillaries has a band structure. The interaction of low order air core modes is extremely small with the cladding at a sufficiently large value of the air core diameter near the centers of the transmission bands ${ }^{15}$. The new type of gasfilled hollow core fiber lasers created on their basis and made of silica glass were devoid of the disadvantages that 
were listed above for fluoride fiber lasers. This led to the creation of sufficiently efficient fiber lasers the principle of operation of which is based on two physical mechanisms, namely, population inversion realized by intrinsic absorption of gas molecules ${ }^{10,16,17}$ (Fig. 1) and the stimulated Raman scattering ${ }^{18,19}$. It should be taken into account that Raman lasers based on the hollow core micro-structured fibers have a threshold which is about 5-6 orders of magnitude higher than that based on the population inversion, which leads to all-reported Raman lasers being pulsed.

In a paper recently published on Light: Science and Applications, the authors set a task of creating a CW fiber laser based on silica glass hollow core negative curvature fiber operating with the highest possible output power at wavelengths greater than $4 \mu \mathrm{m}$ in the broadest tuning range $^{20}$. For these purposes, they used a population inversion realized by intrinsic absorption of $\mathrm{HBr}$ molecules. In their previous work ${ }^{17}$ they used $\mathrm{CO}_{2}$ filled hollow core

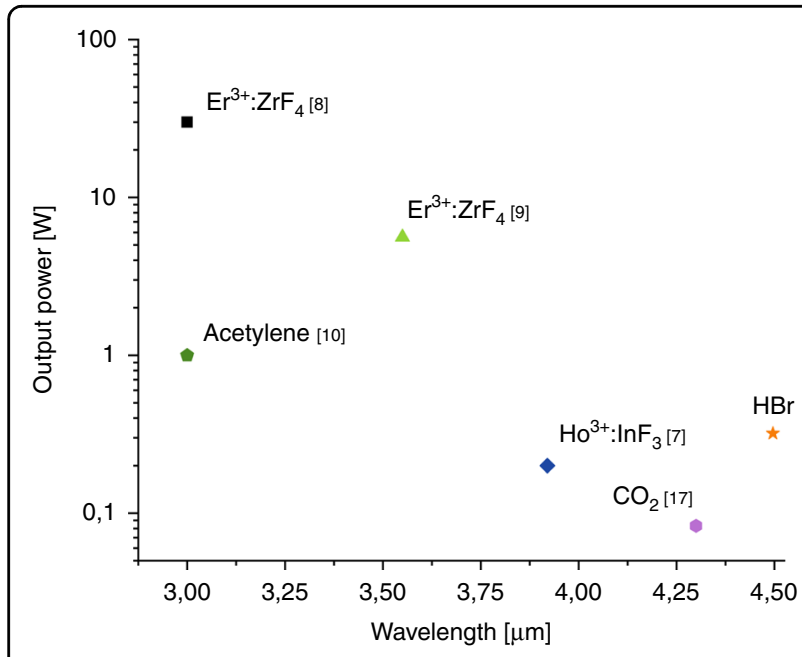

Fig. 1 Continuous wave mid-IR fiber lasers. Summary of the stateof-art continuous wave mid-IR fiber lasers in terms of output and lasing wavelengths. fiber but the possible output laser wavelength range was small enough (Fig. 1) due to the transition properties of $\mathrm{CO}_{2}$ molecules. In their new work, the authors used a narrow linewidth $2 \mu \mathrm{m}$ thulium-doped fiber amplifier seeded by a group of fine-tunable diode lasers to pump a 5meter-long silica glass hollow core fiber with a crosssection similar to the cross-section of the hollow core fiber shown in Fig. 2 (left). The hollow core fiber was filled with low -pressure $\mathrm{HBr}$ gas. The maximum laser output of $500 \mathrm{~mW}$ was achieved at wavelength of $4.2 \mu \mathrm{m}$ with tuning range of $686 \mathrm{~nm}$ when the $\mathrm{HBr}$ pressure was $5 \mathrm{mbar}$. Also, the laser output of about $300 \mathrm{~mW}$ was achieved at the longest wavelength of $4.496 \mu \mathrm{m}$ among $\mathrm{CW}$ fiber lasers (Fig. 1). Further research in this field, according to the authors of the paper, is connected with two main directions, namely, an employment of all-fiber structure coupling with low loss between hollow core fibers and solid- core fibers and also achieving high power output.

The situation can also be changed with creation of the hollow core fibers made of non-silica glasses made of tellurite glasses or chalcogenide glasses ${ }^{21-23}$. Their use will significantly reduce material losses of the fiber cladding in the mid -IR spectral range to obtain CW laser generation at wavelengths greater than $4.5 \mu \mathrm{m}$. To date, tellurite hollow core fibers have been developed by an extrusion and draw approach. $\mathrm{In}^{22}$ the fiber losses of 4.8 and $6.4 \mathrm{~dB} / \mathrm{m}$ were measured at 5.6 and $5.8 \mu \mathrm{m}$, respectively. According to the authors ${ }^{22}$, this gives hope that in the near future it will be possible to fabricate tellurite hollow core optical fibers with sub $1-2 \mathrm{~dB} / \mathrm{m}$ loss anywhere in the technologically important spectral region between 4.5 and $6.5 \mu \mathrm{m}$. $\mathrm{In}^{23}$ the authors reported the fabrication of a hollow core fiber drawn from chalcogenide glass 3D printed preform. This fiber showed several transmission bands in the $2-12 \mu \mathrm{m}$ spectral range.

In conclusion, CW gas fiber lasers based on the new type of hollow core fibers are becoming an emerging competitor to their rare-earth-doped counterparts in the mid-IR spectral range. It is quite possible that in the near
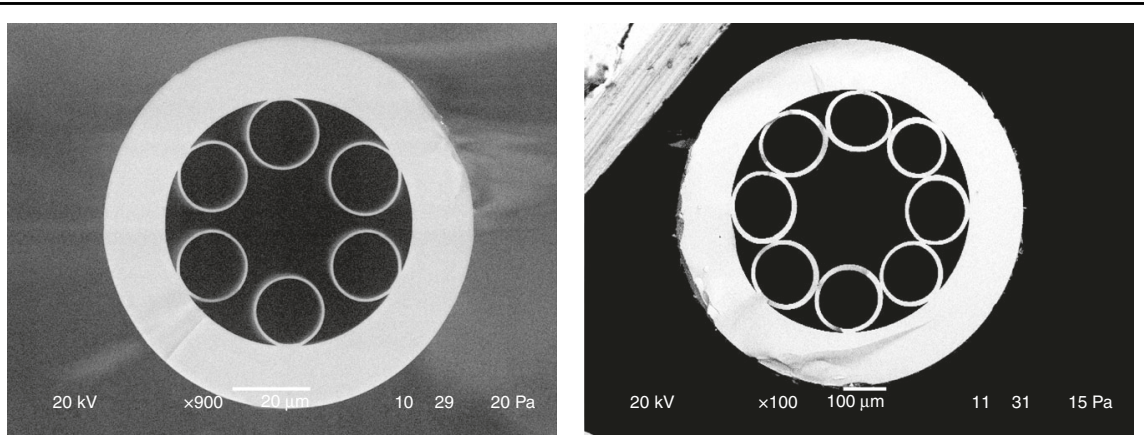

Fig. 2 Cross - sections of the negative curvature hollow core fibers made of silica glass (left) and chalcogenide glass (right). The fibers were fabricated at Dianov Fiber Optics Research Center. 
future they will become a good alternative compared to other types of fiber lasers, for creating CW coherent light sources in the mid-IR spectral range.

\section{Conflict of interest}

The author declares no competing interests.

Published online: 11 February 2022

\section{References}

1. Faist, J. et al. Quantum cascade laser. Science 264, 553-556 (1994).

2. Deloach, L. D. et al. Transition metal-doped zinc chalcogenides: spectroscopy and laser demonstration of a new class of gain media. IEEE J. Quantum Electron. 32, 885-895 (1996).

3. Vodopyanov, K. L. Mid-infrared optical parametric generator with extra-wide (3-19- $\mu \mathrm{m})$ tunability: applications for spectroscopy of two-dimensional electrons in quantum wells. J. Optical Soc. Am. B 16, 1579-1586 (1999).

4. Jackson, S. D. Towards high-power mid-infrared emission from a fibre laser. Nat. Photonics 6, 423-431 (2012).

5. Falconi, M. C., Laneve, D. \& Prudenzano, F. Advances in mid-IR fiber lasers: tellurite, fluoride and chalcogenide. Fibers 5, 23 (2017).

6. Tao, G. M. et al. Infrared fibers. Adv. Opt. Photonics 7, 379-458 (2015).

7. Maes, F. et al. Room-temperature fiber laser at $3.92 \mu \mathrm{m}$. Optica 5, 761-764 (2018).

8. Fortin, V. et al. $30 \mathrm{~W}$ fluoride glass all-fiber laser at $2.94 \mu \mathrm{m}$. Opt. Lett. 40, 2882-2885 (2015)

9. Maes, F. et al. 5.6 W monolithic fiber laser at $3.55 \mu \mathrm{m}$. Opt. Lett. 42, 2054-2057 (2017).
10. Xu, M. R. et al. Continuous-wave mid-infrared gas fiber lasers. IEEE J. Sel. Top Quantum Electron. 24, 0902308 (2018).

11. Pryamikov, A. D. et al. Demonstration of a waveguide regime for a silica hollow-core microstructured optical fiber with a negative curvature of the core boundary in the spectral region $>3.5 \mu \mathrm{m}$. Opt. Express 19, 1441-1448 (2011).

12. Wang, Y. Y. et al. Low loss broadband transmission in hypocycloid-core Kagome hollow-core photonic crystal fiber. Opt. Lett. 36, 669-671 (2011).

13. Yu, F. et al. Low loss silica hollow core fibers for 3-4 $\mu \mathrm{m}$ spectral region. Opt. Express 20, 11153-11158 (2012).

14. Kolyadin, A. N. et al. Light transmission in negative curvature hollow core fiber in extremely high material loss region. Opt. Express 21, 9514-9519 (2013).

15. Debord, B. et al. Hypocycloid-shaped hollow-core photonic crystal fiber Part I: Arc curvature effect on confinement loss. Opt. Express 21 28597-28608 (2013).

16. Aghbolagh, F. B. A. et al. Mid IR hollow core fiber gas laser emitting at $4.6 \mu \mathrm{m}$. Opt. Lett. 44, 383-386 (2019).

17. Cui, Y. L. et al. $4.3 \mu \mathrm{m}$ fiber laser in $\mathrm{CO}_{2}$-filled hollow-core silica fibers. Optica $\mathbf{6}$, 951-954 (2019)

18. Gladyshev, A. V. et al. 4.4- $\mathrm{um}$ Raman laser based on hollow-core silica fibre Quantum Electron. 47, 491-494 (2017).

19. Astapovich, M. S. et al. Watt-level nanosecond 4.42- $\mu$ m Raman laser based on silica fiber. IEEE Photonics Technol. Lett. 31, 78-81 (2019).

20. Zhou, Z. Y. et al. Towards high-power mid-IR light source tunable from 3.8 to $4.5 \mu \mathrm{m}$ by HBr-filled hollow-core silica fibres. Light:: Sci. Appl. 11, 15 (2022).

21. Tong, H. T. et al. Mid-infrared transmission by a tellurite hollow core optical fiber. Opt. Express 27, 30576-30588 (2019).

22. Ventura, A. et al. Extruded tellurite antiresonant hollow core fiber for mid-IR operation. Opt. Express 28, 16542-16553 (2020).

23. Carcreff, J. et al. Mid-infrared hollow core fiber drawn from a 3D printed chalcogenide glass preform. Optical Mater. Express 11, 198-209 (2021) 